\title{
Arteriovenous malformation of the filum terminale: an exceptional case
}

\author{
Lucas Troude Jr., Anthony Melot, MD, Hervé Brunel, PhD, and Pierre-Hugues Roche, PhD \\ Department of Neurosurgery, CHU Nord, Marseille, France
}

Arteriovenous malformations (AVMs) of the spine display a variety of different locations, angioarchitectures, and clinical presentations. The authors describe an exceptional case of a filum terminale AVM that is not described in any classification and discuss the origin and management of this malformation. A 59-year-old woman was admitted in June 2012 for cauda equina syndrome. Magnetic resonance imaging and spinal angiography revealed an AVM of the filum terminale, located below the conus medullaris, fed by the anterior spinal artery. After an unsuccessful attempt to reach the nidus with a microcatheter, the AVM was resected. At 20 months after surgery, the patient was fully independent and radiological images confirmed the exclusion of the malformation. AVMs that originate from the filum terminale are exceptional. According to updated classifications, AVMs of the filum terminale should be categorized as a separate entity.

http://thejns.org/doi/abs/10.3171/2015.3.JNS142446

KEY WORDS arteriovenous malformation; spinal cord; surgery; filum terminale; vascular disorders

$\mathrm{B}$ ROADLY speaking, spinal arteriovenous lesions include arteriovenous malformations (AVMs) and pure fistulas. Recently, a new subtype of AVM that develops at the level of the conus medullaris has been added to the previous classifications. ${ }^{7,8}$ In this paper, we present an original description of an AVM involving the filum terminale, which was cured by resection, and show that this lesion does not fit with previous classifications.

\section{Case Report}

History and Presentation

A 59-year-old woman was initially admitted to a neurosurgical unit in 1997 for progressive cauda equina syndrome. In the past, the patient had experienced chronic alcoholism, posttransfusional hepatitis $\mathrm{C}$, and mixed cirrhosis Child-Pugh Class. Magnetic resonance imaging and spinal angiography revealed an AVM located below the conus medullaris, fed by the anterior spinal artery (ASA) originating from the right T-10 intercostal pedicle. At that time, an attempt at embolization failed, without further intervention, and the patient was then lost to follow-up.

The patient was admitted to our institution in June 2012 with a progressive and severe paraparesis. A clinical examination showed a cauda equina syndrome with bilateral sciatic leg pain and weakness, and a lack of bladder con- trol causing urinary incontinence. She was unable to walk; her motor score was $2 / 5$ for the main muscles of the lower limbs. Sacral cutaneous ulcerations and saddle anesthesia were observed.

The preoperative workup showed the following findings. On MRI, the spinal cord ended immediately below the T12-L1 disc (Fig. 1). Around and below the spinal cord, there was a serpentine pattern of dilated vessels occupying the entire lumbar spinal canal cross-section, with a compact network of low signal corresponding to the nidus.

Digital subtraction angiography (DSA) identified a compact nidus located at the L-1 level, under the conus, fed by the ASA that was dilated (Fig. 2). Two aneurysms were identified within the nidus. Early filling of perimedullary tortuous veins was shown.

Together with the neuroradiologist we planned a combined treatment, starting with a new attempt at embolization followed by a microsurgical resection of the AVM in case of incomplete occlusion. The endovascular step that catheterized the ASA failed to guide the microcatheter to the vicinity of the nidus.

\section{Operation and Postoperative Course}

The surgery was performed using a T12-L2 laminectomy. The dura was opened on the midline under a mi- 


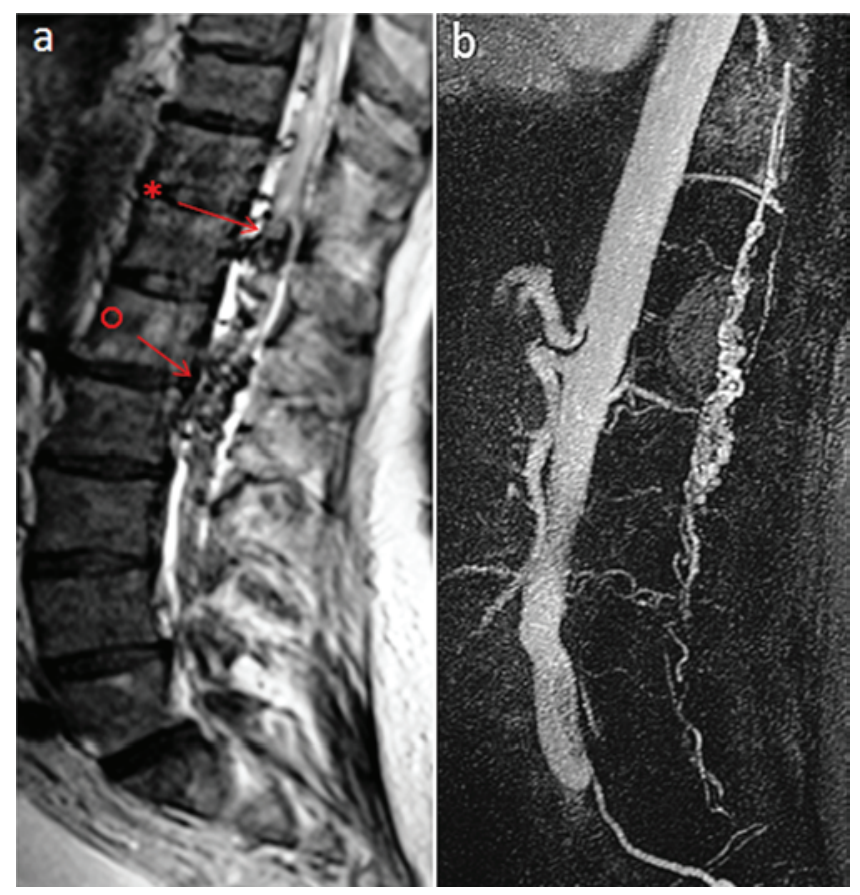

FIG. 1. Preoperative MR images. The spinal cord ended immediately below the T12-L1 disc (a). Around and below the spinal cord, there was a serpentine pattern of dilated vessels occupying the entire lumbar canal cross-section, with a compact network of low void corresponding to the nidus $(\mathrm{a}$ and $\mathbf{b})$. Asterisk = conus medullaris; circle $=$ compact nidus located at the L-1 level.

croscope with preservation of the underlying vessels (Fig. 3 ). An engorgement of the microvasculature of the conus was observed; the sensitive roots were shifted dorsally and laterally due to the bulking of the vascular mass. An at- tempt at direct mobilization of the nidus resulted in copious infield bleeding. Using the preoperative DSA findings we could identify the main feeder on the right side of the AVM and occlude it with a 5-mm Yaşargil clip. This maneuver allowed an instantaneous interruption of the active bleeding and a decreasing of the intranidal pressure. The nidal resection was started at its upper pole and we could observe a clear delineation between the vascular mass and the conus. The nidus was clearly developed from the filum terminale, which was coagulated and divided. The AVM was removed en bloc with final interruption of the main draining vein at the end of the resection. Macroscopically, the lesion displayed the characteristic of a compact nidus that was confirmed later on by the pathologist who analyzed the entire tissue specimen.

During the postoperative course, the patient experienced a significant improvement of her motor deficit as well as of her sphincter dysfunction. The patient was discharged on Day 12 and referred to the rehabilitation center.

Spinal cord MRI conducted 5 months after surgery (Fig. 4a and b) confirmed the lack of residual vessel malformation under the conus and disappearance of the spinal cord edema. At 1 year after surgery another spinal DSA session was performed, including the catheterization of the T8-L3 pedicles. Injection of the right T-10 pedicle did not show the ASA for an unknown reason. The postoperative DSA did not display any residual nidus or early venous filling (Fig. 4c), which meant that the AVM was cured. Two years after surgery the patient was able to walk independently but still complained of urinary disorders and neuropathic pain in the inferior limbs.

\section{Discussion}

Spinal AVMs are rare congenital diseases that can

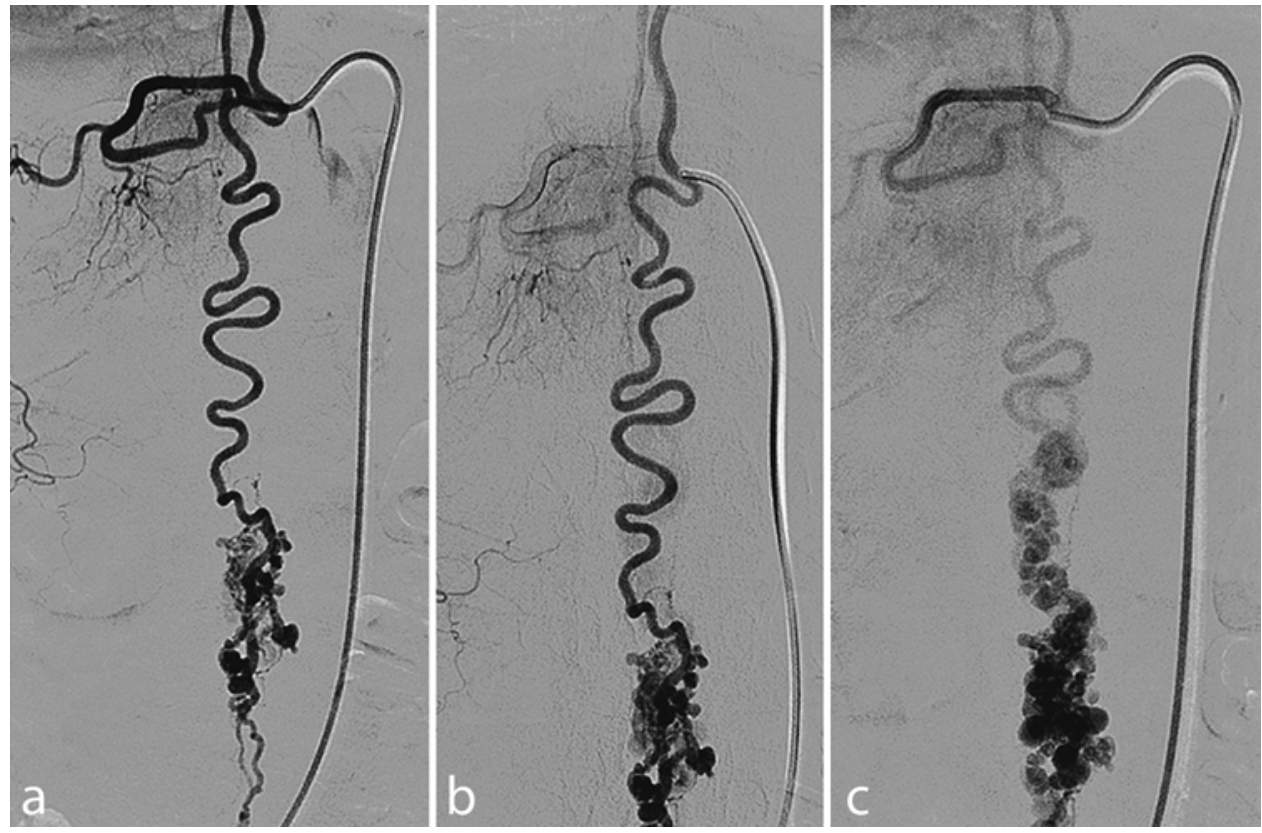

FIG. 2. Preoperative DSA. There was a compact nidus located at the L-1 level, under the conus, fed by the ASA that was dilated. Two aneurysms were identified within the nidus. Early filling of perimedullary tortuous veins was observed. 

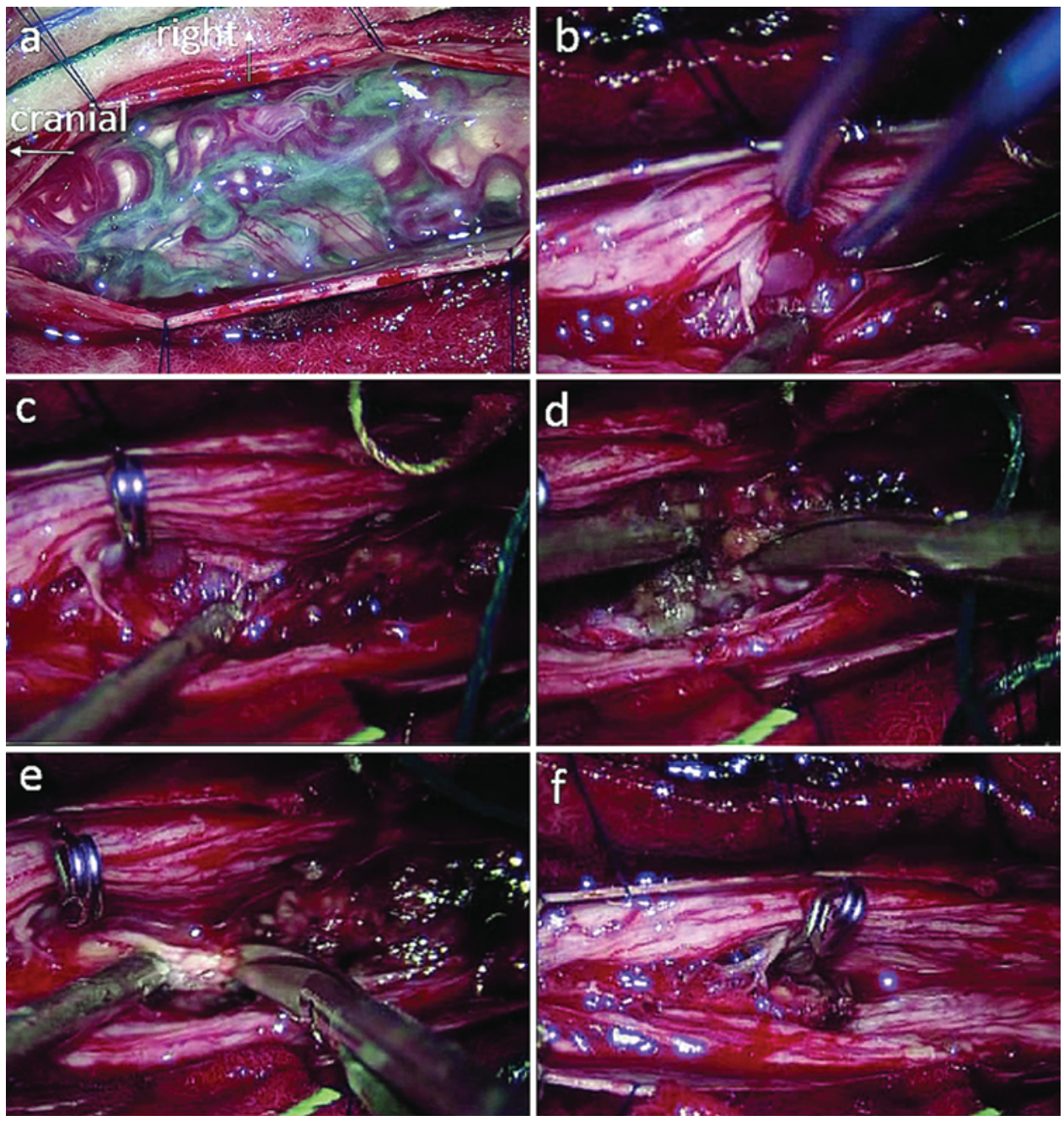

FIG. 3. Perioperative images. a: The dura is opened, showing the general presentation of the AVM under the conus. b: Identification of the main dilated feeder coming from the distal portion of the ASA. c: Placement of a microclip on the main feeder. d: Dissection of the upper pole of the AVM. e: Sectioning of the filum terminale under the conus. f: Final view after en bloc removal of the AVM.

develop irrespective of the level of the cord. Most spinal AVMs are diagnosed before the fourth decade of life. In up to $50 \%$ of cases, patients usually present with a suddenonset severe neurological deficit due to hemorrhages, often partially regressive, and relapse to a bedridden state in 10-20 years. In the other cases, patients experience gradual loss of neurological function, resulting from different mechanisms of cord injury, including medullary venous congestion, ischemia due to vascular steal, or mechanical compression by an aneurysm.

AVMs that originate from the filum terminale are exceptional. To the best of our knowledge, only 1 previous case has been described, in a patient reported by Djindjian et al. ${ }^{3}$ Anatomical and angiographic studies on cadavers indicate that the distribution of the vascularization of the filum appears constant, fed by a single artery (the artery of the filum), arising from one or multiple (network) final and distal branches of the ASA. ${ }^{4}$

AVMs of the filum terminale can be misdiagnosed with 2 distinct lesions: 1) intramedullary AVMs originat- ing from the conus medullaris, ${ }^{8}$ and 2) dural fistulas with serpentine veins located at the level of the filum.

Referring to the classifications of spinal arteriovenous lesions, AVMs of the filum terminale can be classified as intradural AVMs, but no classification individualizes AVMs of the filum terminale as a separate entity. ${ }^{1,2,5-7}$ Many arguments for a new subtype of AVM that does not fit with conus medullaris AVMs are provided by the present case: the clinical presentation, characterized by a gradual peripheral syndrome without myelopathy; the radiological features (nidus apart from the conus, fed by the terminal branch of the ASA, which is the usual feeder of the filum); and the operative findings (lack of conus involvement, need to cut the filum to mobilize the upper pole of the nidus).

Regarding therapeutic management, it is now well established that spinal cord AVMs justify a first-step embolization, optionally followed by complete resection of the nidus in case of incomplete endovascular treatment. ${ }^{6,8}$ Due to the rarity of a filum terminale AVM, there is no 

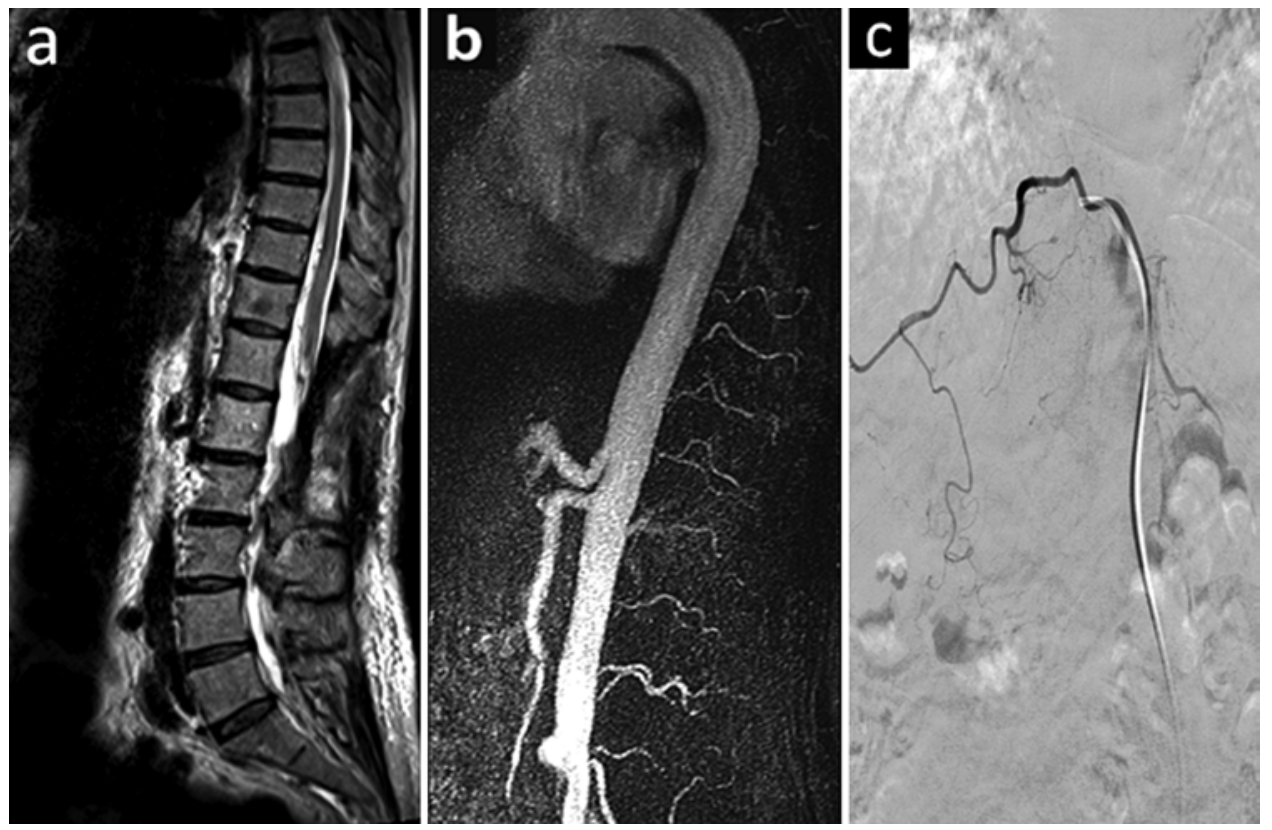

FIG. 4. Postoperative images. MR image of the conus medullaris showing no residue of the AVM. The spinal cord edema has disappeared (a). No more vessels are under the conus. Postoperative MR angiography (b) and angiography (c) showing no residue of the AVM after injection of the right T-10 pedicle.

therapeutic guideline to adopt, but one may assume that the same sequence of procedures is the best option. Use of endovascular techniques can take advantage of new advances in microcatheters and the use of Onyx (ev3). However, perinidal or nidal occlusions look technically difficult and can involve risk due to the need to navigate distally through the ASA. Surgery allows an immediate total exclusion of the lesion in a single session (1-step treatment) and nerve root decompression that promotes early recovery of the deficit. In the present case, the challenge was to find the main feeder early after dural opening and to preserve the main draining veins that ran dorsally. Lesions of the filum terminale are very accessible to surgery, contrary to AVMs of the conus medullaris, probably with a much better functional prognosis; this is why it appears relevant to distinguish these two entities to select the best therapeutic options.

\section{References}

1. Dashti SR, Kim LJ, Kakarla UK, Park MS, Stiefel MF, Spetzler RF: Spinal arteriovenous malformations, in Mohr JP, Wolf PA, Grotta JC, et al (eds): Stroke, ed 5. Philadelphia: Elsevier, 1366-1373, 2011

2. Dashti SR, Toledo M, Kim LJ, Spetzler RF: Classification of spinal arteriovenous lesions: arteriovenous fistulas and arteriovenous malformations, in Winn HR (ed): Youman's Neurological Surgery, ed 6. Philadelphia: Elsevier, 2011, pp 4146-4152

3. Djindjian M, Djindjian R, Rey A, Hurth M, Houdart R: Intradural extramedullary spinal arterio-venous malformations fed by the anterior spinal artery. Surg Neurol 8:85-93, 1977

4. Djindjian M, Ribeiro A, Ortega E, Gaston A, Poirier J: The normal vascularization of the intradural filum terminale in man. Surg Radiol Anat 10:201-209, 1988
5. Kim LJ, Spetzler RF: Classification and surgical management of spinal arteriovenous lesions: arteriovenous fistulae and arteriovenous malformations. Neurosurgery $59(5$ Suppl 3):S195-S201, S3-S13, 2006

6. Riina HA, Spetzler RF: Classification of vascular lesions affecting the spinal cord. Operative Tech Neurosurg 6:106115,2003

7. Spetzler RF, Detwiler PW, Riina HA, Porter RW: Modified classification of spinal cord vascular lesions. J Neurosurg 96 (2 Suppl):145-156, 2002

8. Wilson DA, Abla AA, Uschold TD, McDougall CG, Albuquerque FC, Spetzler RF: Multimodality treatment of conus medullaris arteriovenous malformations: 2 decades of experience with combined endovascular and microsurgical treatments. Neurosurgery 71:100-108, 2012

\section{Disclosure}

The authors report no conflict of interest concerning the materials or methods used in this study or the findings specified in this paper.

\section{Author Contributions}

Conception and design: Troude. Acquisition of data: Troude. Analysis and interpretation of data: Troude. Drafting the article: Troude. Critically revising the article: Melot, Brunel, Roche. Reviewed submitted version of manuscript: all authors. Approved the final version of the manuscript on behalf of all authors:

Troude. Administrative/technical/material support: Brunel. Study supervision: Roche.

\section{Correspondence}

Lucas Troude, 20 Rue Marengo, Marseille 13006, France. email: lucas.troude@hotmail.fr. 